\title{
Team-process Factors for Team Creativity in Taiwan
}

\author{
Yeh Yu-Mei ${ }^{1, *}$, Li Feng-Chia $^{2}$, Lin Hung-Yuan ${ }^{3}$ \\ ${ }^{1}$ Department of Marketing and Distribution Management, Hsing Wu University, Taiwan \\ ${ }^{2}$ Department of Distribution Management, National Chin-Yi University of Technology, Taiwan \\ ${ }^{3}$ Department of Information Management, Shih Hsin University, Taiwan
}

Copyright $\subseteq 2017$ by authors, all rights reserved. Authors agree that this article remains permanently open access under the terms of the Creative Commons Attribution License 4.0 International License

\begin{abstract}
With the globalization of markets, to remain competitive, companies require not only new product ideas and superior approaches for responding to customer needs but also improved processes or environments in the workplace. Firms cannot rely on a single worker's creativity to implement innovation successfully but must encourage collective creativity, which focuses on collaboration and joint success. This study focused on attaining a more profound understanding of creativity theory as developed from the individual to team levels and on the team process of how members contribute by combing relevant inputs by using an optimal method for yielding a creative team response. Following the model of team creativity of Dzindolet and Paulus, we identified crucial team member variables and analyzed the effects of these variables on interactions among cognitive, social, and motivational processes and on the enhancement of team creativity. Task proficiency, collectivistic value orientation, and team vitality were determined to be three team member variables crucial for team creativity. The structural equation modeling (SEM) analyses were also used to show that team vitality mediates the positive relationships among task proficiency, collectivistic value orientation, and team creativity. In study, we also discuss the implications of these results for teaching research and practice.
\end{abstract}

Keywords Team Task Proficiency, Collectivistic Value Orientation, Team Vitality, Team Creativity

\section{Introduction}

With the globalization of markets, technological changes and production cycles have accelerated rapidly. This environment has heightened business competition worldwide [7]. The work environment requires greater ingenuity and more fresh ideas from workers [10], and firms demand more creativity and innovation from their employees [26]. Consequently, focus has increasingly been placed on creativity and innovation, and corporations' increasingly emphasize the need for creativity and innovation in their organizations and processes [17] [31] [35].

The Taiwanese economic system is primarily based on small and medium enterprises [37]. Small and medium enterprises in Taiwan need to have more flexibility and innovation to strengthen their competitiveness. By the historical and cultural background, Taiwan and Japan have maintained friendly economic cooperation. Taiwanese companies learn from Japanese companies a lot of business model, innovative culture and quality management. Therefore, employers emphasize employees' intangible development of team creativity and creative behaviors in Taiwan [40] [41].

Creativity is a driver for innovation. Amabile and coworkers defined creativity as the production of novel and useful ideas in any domain [2] [30]. Most firms cannot rely on a single worker's creativity to implement innovation successfully; they must combine the creativity of all of the workers to achieve innovation. Thus, collective creativity that focuses on collaboration and joint success may be preferable [8] [7] [39].

Although scholars have gained much knowledge on how to enhance team, group, and organizational creativity, much knowledge remains to be learned. Gaps persist in the literature, and the need for a more comprehensive understanding regarding how to enhance team, group, and organizational creativity remains. Few studies have examined specific organizations or teams. A clear framework for a theoretical understanding of creativity and the accompanying empirical studies are lacking [1] [29]. Relevant research has focused on organizational settings (e.g., [34]), but studies examining the implications of this research are required [17]. To address the mentioned gaps in team creativity research, this study focused on attaining a more comprehensive understanding of creativity theory as developed from the individual to team levels and on the process of how team members contribute by combing relevant inputs by using an optimal method for yielding a creative team response [4] [29].

Paulus and coworkers have made considerable progress 
in studying the social and cognitive influences of the team creative process but think that many unresolved questions require additional research. Hence, according to social influence approach, this study followed the model of team creativity of Dzindolet and Paulus [17] to identify crucial team member variables and analyze the effects of these variables on interactions among cognitive, social, and motivational processes and on the enhancement of team creativity. Amabile [1] emphasized that creativity does not occur spontaneously or randomly but occurs when the appropriate combinations of knowledge, skills, and motivation enable an individual to create new ideas. Team members' degree of familiarity with the knowledge and skills related to their task and motivation affect the team's creativity. Therefore, this study began with an exploration of how the team member variables task proficiency and vitality (reflecting an energetic force), which initiate intrinsic motivation (i.e., psychological forces that stimulate behavior), contribute to team creativity. In addition, the collectivistic values of a team are highly conducive to team creativity [7]. In the social-motivational processes of a team are defined as processes in which team members coordinate the direction, intensity, and persistence of their efforts to achieve their collective goals [11] [36]. When team members consciously adopt the team's collective values during the social process, team motivation during the motivational process is enhanced. Therefore, the study used the team member variable "collectivistic value orientation" to investigate the relationships among team task proficiency, vitality, and team creativity and, thus, determine whether other crucial team member variables exist and how these variables enhance team creativity by affecting the interactions among cognitive, social, and motivational processes. This study developed a clear conceptual model and logic of how task proficiency, collectivistic value orientation, and vitality enable creativity at collective levels and addresses the lack of a clear theoretical framework of and empirical studies on team creativity.

\section{Theoretical Background and Hypothesis}

\subsection{Relationship between Team Task Proficiency and Collectivistic Value Orientation}

Teams are often assembled to engage in multifaceted and complex endeavors. Thus, team tasks provide a range of knowledge-intensive challenges for team members [4]. Team members must have task-relevant knowledge and skills to generate ideas and solutions to the team's problems.

Marks, Mathieu, and Zaccaro defined the team process as "members' interdependent acts that convert inputs to outcomes through cognitive, verbal, and behavioral activities directed toward organizing task work to achieve collective goals" (as cited in [22]). The manner in which team members organize and execute their tasks affects the team's collective goals. They can use these knowledge resources related to the team's collective goals to recombine existing assets and improvise in the moment to enhance outcomes and to construct a more integrated picture of their work situation and more effectively apply their skills [33]. As individuals understand how to recombine this existing knowledge related to the team's collective goals into new techniques for problem solving, they perceive how their work fits into the larger scheme and, thus, are likely to relate more attentively with others [16].This study proposed the following Hypothesis 1: Team task proficiency is positively associated with collectivistic value orientation.

\subsection{Relationship between Collectivistic Value Orientation and Team Creativity}

Creativity at work refers to the creation of novel and useful products, services, ideas, procedures, or processes [1] [22] [36]. Most creative acts occur in a collaborative context [38]. Because of the creative individuals embedded in an organization or group context, many organizations now emphasize performance at the team level [4]. The high level of individualism has a positive influence on the creativity of society [32]. Collectivism is rather important for realization of an idea (innovation, project performance) [32]. The team creativity model of Dzindolet and Paulus posits that team-level inputs, such as team member characteristics, team structure, and team climate, influence team creativity through the intervening mechanisms of social, cognitive, and motivational processes [36].

After the cognitive process, members adopt the team's view and create a shared understanding, guiding team processes and output more effectively [13] [36]. Teams with members collaborating closely to achieve common goals are more creative than groups without such members [7] [39].The collectivistic values of a team greatly benefit team creativity and group performance [7].An emphasis on the team process is critical because there is strong support that team processes are positively associated with innovation [21]. We proposed the following Hypothesis 2: Collectivistic value orientation benefits team creativity.

\subsection{Relationship between Team Vitality and Team Creativity}

Carmeli and Spreitzer [10] showed that vitality facilitates employee involvement in creative work. When individuals experience vitality in their work, they are likely to have the energy and motivation to engage in innovative work.

Vitality inspires individuals' recognition, intrinsic motivation, and achievement, causing them to become passionate about their work content and work relationships [10]. Vitality is a type of positive mood [19]. It may indicate a person's level of intrinsic motivation, which has 
been found to be a vital prerequisite for innovative behavior [10].Thus, we proposed the following Hypothesis3: Team vitality is positively associated with team creativity.

\subsection{Relationships among Team Task Proficiency, Collectivistic Value Orientations, Team Vitality, and Team Creativity}

According to the team creativity framework of Dzindolet and Paulus [17], group, task, and situational variables interact and affect creative outputs through motivational, cognitive, and social processes. Therefore, task proficiency of task variable to promote creative output it must interact with motivational, cognitive, and social processes.

According to this team creativity model, the individual cognitive process associates with the social-motivational process[36].The social-motivational process involves team members coordinating the direction, intensity, and persistence of their efforts to achieve team energy [11][36]. In this highly motivated situation, team members have a positive feeling of having energy available [14].Therefore, this study proposed the following Hypothesis 4: Collectivistic value orientation mediates the positive relationship between team task proficiency and team vitality.

Team creativity requires team members' collective efforts toward the generation of creative ideas or products. When team members consciously adopt collective team values during the social process, team motivation is enhanced during the motivational process and becomes pivotal in workplace creativity [1] [36]. High motivation among team members is a prerequisite for collectivistic value orientation to result in high team creativity [7]. Team members must be motivated to apply themselves to the creative task at hand to benefit from the originality-enhancing effects of collectivistic value orientation [7] [6].In situations requiring high motivation, team members feel the psychological forces and are pushed to perform energized behaviors autonomously (e.g., [25] [26].) Therefore, this study proposed the following Hypothesis 5: Team vitality mediates the positive relationship between collectivistic value orientation and team creativity.

\section{Methods}

\subsection{Sample and Data Collection Procedures}

In this study, we examined 85 creative marketing task teams consisting of a total of 338 third-year university students and led by professors at science and technology universities in Taiwan. These universities focus on cultivating the professional functions required of talented personnel in the service industries, such as retail and wholesale, franchising, information services, and tourism and leisure. We randomly selected 10 science and technology universities before choosing faculties (i.e., business administration, information management and communication, and tourism and hospitality) within these universities, from which five professors who guided student projects were randomly selected. We discussed the research purposes and design of the students' creative marketing projects with these professors in advance. Each team was required to devise creative marketing tasks, which included planning a creative marketing task, generating and revising novel and useful ideas for the task, developing new procedures for the task, performing useful methods pertaining to task difficulties, and formulating ideas for overcoming task difficulties, and finalizing the task. Then, we personally distributed questionnaires and explained the research projects to the professors. Before the semester ended and after the creative marketing task was completed, the professors distributed the questionnaires to the members of the student teams. The professors and external collaborating industry supervisors assessed the creativity of each team; we personally retrieved the assessment results to reduce evaluation apprehension among the subjects and avoid social desirability bias [27]. A total of 346 questionnaires from 85 teams were recovered; eight were invalid, yielding 338 valid questionnaires with a valid recovery rate of $34 \%$. The average size of the 85 teams was 5.8 members $(\mathrm{SD}=2.4)$, and the team size ranged from 3 to 5 members. The average response rate for the participating teams was $72.0 \% \quad(\mathrm{SD}=11.7)$. The domains of the participating teams varied among business administration (BA $=78.1 \%)$, information management $(\mathrm{IM}=83.5 \%)$, and communication, tourism, and hospitality $(\mathrm{CTH}=72.2 \%)$.

\subsection{Analyses}

We performed confirmatory factor analyses (CFAs) to investigate the discriminant validity of the factor structures of task proficiency, collectivistic value orientation, team vitality, and team creativity. The overall model fit was assessed using the comparative fit index (CFI), the incremental fit index (IFI), and the root mean square error of approximation (RMSEA; [3]). In addition, because we measured four variables at the team level, we conducted an analysis of the level of agreement among team members for each construct. When interrater agreement $\left(r_{w g}\right)$ was greater than .70, we analyzed the sum of the individual data (Demaree, James, \& Wolf, 1993). To test intergroup variability, we compared median $r_{w g}$ scores and intraclass correlation coefficients: ICC [1] and ICC [2]. ICC [1] values near or exceeding .12 indicated a desirable level, and ICC [2] values near or exceeding .60 confirmed the validity of the hierarchical constructs (James et al., 1993).

\section{Measurements}

To prevent ambiguity and uncertainty in the respondents' 
answers, we used a 6-point Likert-type scale for all of our measurements, with 1 representing strongly disagree (or extremely low) and 6 representing strongly agree (or extremely high). We designed two questionnaires, a questionnaire for the professors and collaborating industry supervisors (i.e., external experts) and a questionnaire for the students. All of the students were required to complete team task proficiency, collectivistic value orientation, and team vitality scales, and the professors and external experts completed a team creativity scale. Because of the cross-sectional nature of the data, the raw data on the variables were obtained from various sources and several procedures were used to reduce common method variance [27]. First, we collected the independent variable at one point in time and both the mediator and the dependent variables at another point in time with a lag of twelve weeks between Time 1 and Time 2. This reduced the potential bias associated with collecting data at one point in time. Second, because the mediator (team vitality) and the dependent variable (team creativity) were collected at the same point in time, we used a CFA to compare two models: (a) a one-factor model in which all of the observed items of both the mediator variable and the dependent variable were loaded onto one latent variable and (b) a two-factor solution in which all of the observed items of the mediator variable were specified to one latent variable and all of the observed items of the dependent variable were specified to another latent variable.

The results of the one-factor solution yielded the following fit indices: $\chi^{2}=690$ with 112 degrees of freedom (df); $\mathrm{CFI}=.79$; IFI=.80; TLI=.72; and RMSEA=.16. The results of the two-factor model showed a more favorable fit with the data, yielding the following fit indices: $\chi^{2}=272.1$ with $\quad$ df $=111 ; \quad$ CFI $=.94 ; \quad$ IFI $=.94 ; \quad$ TLI $=.92 ; \quad$ and RMSEA $=.08$. These results suggest that potential biases in this study associated with collecting the data for the mediator and dependent variables from a single source at one point in time were not likely to be severe. In addition, we employed an adapted backward translation procedure for all of the scales, with three scholars subsequently reviewing the translations and revisions to ensure the suitability of the items for our subjects and the content validity of the scales.

\subsection{Team Task Proficiency}

This study adopted two items revised by Armenakis et al. (2006). The teams' proficiency in problem solving was scored as a function of "My team successfully solved problems and team-related tasks". High scores indicated a high level of team task proficiency. We summed and averaged the two items, and Cronbach's $\alpha=.86, r_{\mathrm{wg}(\mathrm{i})}=.84$, $\operatorname{ICC}(1)=.118, \operatorname{ICC}(2)=.64, F=127.54$, and $p<.005$.

\subsection{Collectivistic Value Orientation}

The study adopted two items developed by Bechtoldt et al.
(2010). Therefore, to evaluate the adequacy of our collectivistic value orientation, the students were asked to report the extent to which they agreed with the following two items: "I and my team members have tried to achieve the best possible outcome for this team" We summed and averaged the two items; Cronbach's $\alpha=.78, r_{\mathrm{wg}(\mathrm{j})}=.89, \operatorname{ICC}(1)=.145$, $\operatorname{ICC}(2)=.51, F=98.26$, and $p<.05$.

\subsection{Team Vitality}

This study adopted five items developed and revised by [5] and applied by [10]. High scores indicated a high level of team vitality. The scale included items such as "My team felt active and energetic in achieving ourwork mission." We summed and averaged the five items; Cronbach's $\alpha=.88$, $r_{\mathrm{wg}(\mathrm{j})}=.86, \operatorname{ICC}(1)=.142, \operatorname{ICC}(2)=.69, F=138.56$, and $p$ $<.001$.

\subsection{Team Creativity}

This study adopted four items developed by [20] and applied by [12]. We summed the averages of the scores for the four assessment items for each team provided by the team professors and the collaborating industry supervisors to measure team creativity. High scores indicated a high level of team creativity. The scale included items such as "This team used novel and feasible ideas to solve team problems." Cronbach's $\alpha=.87, r_{\mathrm{wg}(\mathrm{j})}=0.81, \operatorname{ICC}(1)=.115$, $\operatorname{ICC}(2)=.63, F=125.53$, and $p<.005$.

\subsection{Background Variables}

Teams of university students were the subjects of this study. Such teams differ from the professional teams found in other types of organization. Previous studies have performed team-level analyses, determining that team size and characteristics influence group cohesion [7]. We used a limited design involving the analysis of university characteristics (science and technology universities), student year (third-year students), team size (three to five members per team), team task content (creative marketing planning and implementation), and team grouping (members drew lots to form groups) to address the interactions among the four variables. These variables were the faculties in which the students studied and the gender composition of the teams (all men, all women, or a mix of both).

\section{Results}

To assess the discriminant validity of the study measures, we conducted a CFA of the items regarding collectivistic value orientation, team task proficiency, and team vitality. The hypothesized three-factor model (Model 0) fit the data well: $\chi^{2}(d f=129)=339.7$, CFI $=.95$, RMSEA $=.07$. This measurement model was compared with two two-factor 
models (Models 1 and 2) in which collectivistic value orientation was merged with either team task proficiency or team vitality into a single factor. As presented in Table 1, the hypothesized three-factor model demonstrated a significantly better fit than did the alternative models
(Model 1, $\Delta \chi^{2}=23.2$ and $p<.001 ;$ Model 2, $\Delta \chi^{2}=69.7$ and $p<.001$ ). These results indicated that the three constructs were empirically distinct and that the scales measuring the constructs had sufficient discriminant validity.

Table 1. Results of the Confirmatory Factor Analysis and Chi-Square Difference Tests

\begin{tabular}{|c|c|c|c|c|c|c|c|c|c|}
\hline Measurement models & $x^{2}$ & $\mathrm{df}$ & CFI & AIC & AGFI & TLI & RMSEA & $\Delta \chi^{2}$ & P value of $\Delta \chi^{2}$ \\
\hline $\begin{array}{c}\text { Model 0: Hypothesized } \\
\text { three-factor model }\end{array}$ & 339.7 & 129 & .95 & 423.77 & .86 & .94 & .07 & $---{ }^{--}$ & \\
\hline $\begin{array}{c}\text { Model 1: Two-factor } \\
\text { model(Combining TTP and } \\
\text { CVO into a single factor) }\end{array}$ & 362.9 & 132 & .94 & 440.86 & .85 & .93 & .07 & 23.2 & $<.001$ \\
\hline $\begin{array}{c}\text { Model 2: Two-factor } \\
\text { model(Combining CVO and } \\
\text { TV into a single factor) }\end{array}$ & 409.41 & 132 & .93 & 487.41 & .83 & .92 & .08 & 69.7 & $<.001$ \\
\hline
\end{tabular}

Note. $\mathrm{CFI}=$ comparative fit index; AIC = Akaike's information criterion; AGFI=Adjusted goodness-of-fit index; TLI= Tucker-Lewis index; RMSEA = root mean square error of approximation; TTP = team task proficiency; $\mathrm{CVO}=$ collectivistic value orientation; and $\mathrm{TV}=$ team vitality.

Table 2. Descriptive Statistics and Inter-correlations $(\mathrm{N}=85)$

\begin{tabular}{|c|c|c|c|c|c|c|c|c|c|c|}
\hline Team-level variables & $M$ & $S D$ & 1 & 2 & 3 & 4 & 5 & 6 & 7 & 8 \\
\hline 1.Team size & 3.97 & 1.32 & 1.00 & & & & & & & \\
\hline 2.Team type-BA & 4.13 & .93 & $.542^{* *}$ & 1.00 & & & & & & \\
\hline 3.Team type-IM & 4.22 & 1.16 & $.626^{* *}$ & $519^{* *}$ & 1.00 & & & & & \\
\hline 4.Team type-CTH & 4.08 & .91 & $.538^{* *}$ & $463^{* *}$ & $725^{* *}$ & 1.00 & & & & \\
\hline 5.Team task proficiency & 4.36 & .74 & $617^{* *}$ & $552^{* *}$ & $677^{* *}$ & $518^{* *}$ & 1.00 & & & \\
\hline 6.Collectivistic value orientation & 4.39 & .75 & $633^{* *}$ & $717^{* *}$ & $593^{* *}$ & $721^{* *}$ & $.899^{* *}$ & 1.00 & & \\
\hline 7.Team vitality & 4.27 & .73 & $525^{* *}$ & $639^{* *}$ & $701^{* *}$ & $649^{* *}$ & $.661^{* *}$ & $.595^{* *}$ & 1.00 & \\
\hline 8.Team creativity & 4.41 & .75 & $603^{* *}$ & $548^{* *}$ & $545^{* *}$ & $537^{* *}$ & $.632^{* *}$ & $.581^{* *}$ & $.776^{* *}$ & 1.00 \\
\hline
\end{tabular}

${ }^{*} \mathrm{p}<.05 .^{* *} \mathrm{p}<.01 .^{* * *} \mathrm{p}<.001$. 


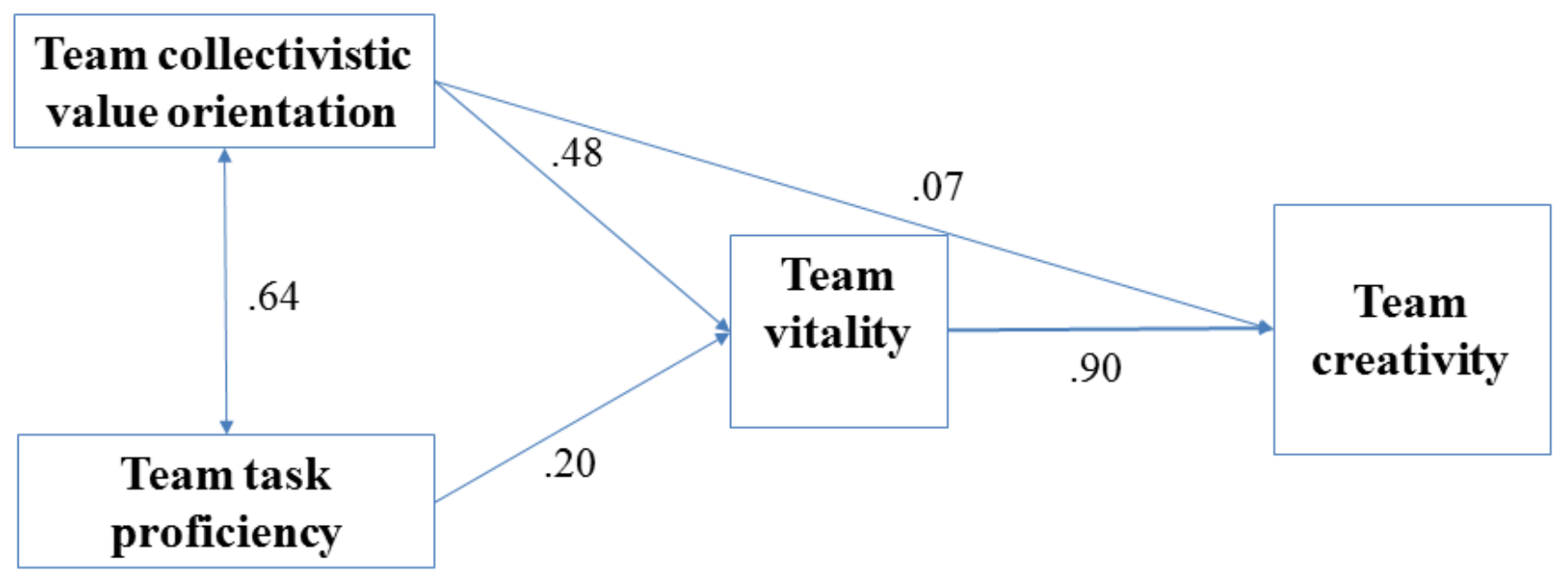

Figure 1. The Hypothesized Four-Factor Model

The descriptive statistics and correlations among the study variables are shown in Table 2 . Collectivistic value orientation was positively correlated with team task proficiency, team vitality, and team creativity. These results are consistent with the relationships proposed in previous sections.

\subsection{Comparison of the Hypothesized Model with the Alternative Models}

A series of structural equation modeling (SEM) analyses were used to test the hypothesized structural model. Hancock and Nevitt [24] suggested that to obtain robust parameter estimates in the SEM, the subject-to-parameter ratio should exceed 2:1. For this study, a full measurement model constructed using all scale items yielded a ratio of 2.7:1 (85 teams: 31 parameters). Previous studies have shown that using single indicators in SEM analyses, which is a common practice (e.g., [36]) yields results comparable to those obtained using multiple indicators (Bennett, Lawrence, Liang, \& Whitelaw, 1990). Consistent with these findings, the results of our study, which were based on single indicators, exhibited patterns identical to those based on multiple indicators.
To assess the hypothesized structural model, we developed a mediation model (Model 0) containing paths from team task proficiency and collectivistic value orientation to team creativity through team vitality. As depicted in Table 3, the proposed mediation model fit the data well in an absolute sense $\left(\chi^{2} / \mathrm{df}=10.60\right.$, GFI $=.87$, $\mathrm{AGFI}=.88, \mathrm{IFI}=.89, \mathrm{RMSEA}=.04$, Akaike's information criterion $(\mathrm{AIC})=697.78, p<.001)$. In addition, we specified four alternative full structural models (Models 1 to 4). The hypothesized four-factor model demonstrated a significantly better fit than did any of the alternative models (Model 1, $\mathrm{GFI}=.79$, AGFI $=.56$, IFI $=.76$, RMSEA $=.29$, $\mathrm{AIC}=555.53, \Delta \chi^{2}=118.2$, and $p<.01 ;$ Model $2, \mathrm{GFI}=.84$, $\mathrm{AGFI}=.81, \mathrm{IFI}=.87, \mathrm{RMSEA}=.06, \mathrm{AIC}=595.4$, $\Delta \chi^{2}=2.59$, and $p<.01 ;$ Model $3, \mathrm{GFI}=.75, \mathrm{AGFI}=.63$, IFI $=.73$, RMSEA $=.21, \mathrm{AIC}=828.4, \Delta \chi^{2}=332.7$, and $p<.05$; and Model 4,GFI $=.76$, AGFI $=.65$, IFI $=.73$, $\mathrm{RMSEA}=.21, \mathrm{AIC}=930.5, \Delta \chi^{2}=336.7$, and $p<.05$. Model 0 was superior to Model 1, Model 2, Model 3, and Model 4 because it was more parsimonious (i.e., lower AIC) and exhibited a better fit to the data(i.e., higher GFI, AGFI, and IFI). Therefore, Model 0 was the model with the best fit. The path coefficients of Model 0 are presented in Figure1. 
Table 3. Comparison of Alternative Structural Models

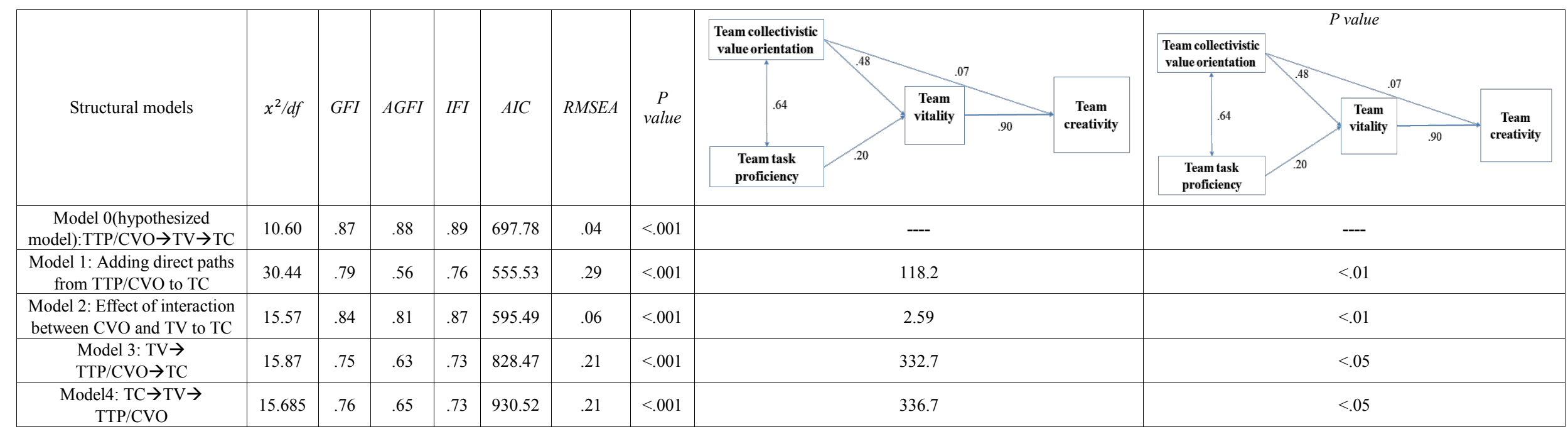

Note. GFI= goodness-of-fit index; AGFI= adjusted goodness-of-fit index; IFI= incremental fit index; AIC= Akaike's information criterion; RMSEA = root mean square error of approximation 
Table 4. Tests of the Indirect Effects

\begin{tabular}{|c|c|c|c|c|c|}
\hline \multirow{2}{*}{ Indirect effects } & \multicolumn{2}{|c|}{ Product of coefficients } & \multicolumn{2}{c|}{$\begin{array}{c}\text { Bootstrapping } \\
\text { bias-corrected 95\% CI }\end{array}$} \\
\hline & Point estimate & SE & P & LL & UL \\
\hline $\mathrm{TTP} \rightarrow \mathrm{CVO} \rightarrow \mathrm{TV}$ & .194 & .397 & .02 & .305 & .593 \\
\hline $\mathrm{CVO} \rightarrow \mathrm{TV} \rightarrow \mathrm{TC}$ & .059 & .068 & .01 & .705 & .824 \\
\hline
\end{tabular}

Note. $\mathrm{CI}=$ confidence interval; $\mathrm{LL}=$ lower limit; $\mathrm{UL}=$ upper limit; Bootstrap sample size $=1000 ; \mathrm{TTP}=$ team task proficiency; $\mathrm{CVO}=$ collectivistic value orientation; TV = team vitality; TC = team creativity.

\subsection{Tests of the Hypotheses}

Hypothesis 1 predicted that team task proficiency is positively related to collectivistic value orientation. SEM analysis was conducted to test the direct effect of team task proficiency and indicated that it had a significant, positive relationship with collectivistic value orientation $(\beta=.64$, $p<.001$ ); thus, Hypothesis 1 was supported. As shown in Figure 1,collectivistic value orientation had a direct effect on team creativity $(\beta=.07, p<.001)$, and team vitality had a highly positively effect on team creativity $(\beta=.90$, $p<.001$ ), lending support to Hypotheses 2 and 3.In mediating effects, first, team task proficiency had a direct effect on collectivistic value orientation $(\beta=.64, p<.001)$. Collectivistic value orientation had a direct effect on team vitality $(\beta=.48, p<.001)$, and consequently, the indirect effect of team task proficiency on team vitality through collectivistic value orientation $(\beta=.64 \times .48=.31, p<.001)$ was superior to the direct effect of team task proficiency on team vitality $(\beta=.20, p<.001)$. Thus, collectivistic value orientation played a significant mediating role. Second, Collectivistic value orientation had direct effects on team vitality $(\beta=.48, p<.001)$, and team vitality positively affected team creativity $(\beta=.90, p<.001)$. Thus, the indirect effect of collectivistic value orientation on team creativity through team vitality was a significantly superior effect $\left(\beta=.48^{*} .90=.43, p<.001\right)$ on the relationship between collectivistic value orientation and team creativity than the direct effect $(\beta=.07, p<.001)$. Third, the proposed mediating effects were validated using the product-of-coefficient approach (Fairchild, Fritz, \& MacKinnon, 2007). The statistical significance of the indirect effect of collectivistic value orientation on team vitality and the indirect effect of team vitality on team creativity were assessed using the bootstrapping procedure (Fairchild et al., 2007). As shown in Table 4, team task proficiency exerted a significant indirect effect on team vitality through collectivistic value orientation (point estimate $=.194, p<0.05$, confidence interval $=.305,1.593$ ). However, collectivistic value orientation exerted a significant indirect effect on team creativity through team vitality (point estimate $=.059, p<0.01$, confidence interval $=.705, .824)$. These results, coupled with the results of the SEM analyses, provide support for Hypotheses 4 and 5.

\section{Discussion}

The findings of this study provide several crucial discussions for research on team creativity and team processes. First, this study clearly demonstrate that team task proficiency, collectivistic value orientation, and team vitality shared in a team contributes to the creativity of the team, supporting previous studies that have asserted that these three variables and team creativity are positively associated. These findings also support the core argument regarding team creativity model of [17].

This study contributed to the discussion of task-relevant knowledge and skills, intrinsic motivation, and sharing ideas to form team value in a team's motivational, cognitive, and social processes. Our findings contribute to the literature on the vitality and relational mechanisms of team creativity. The findings indicate that task proficiency, value orientation, and vitality have a positive impact not only on individuals' cognitions, motivations, and social interactions but also on the collective dynamics operating in a team.

Second, this study's findings provide empirical support for the team creativity model of [17], which models social, cognitive, and motivational processes as a mediating process between team level inputs and team creativity, because the high level of individualism has a positive influence on the creativity of society [32]. Collectivism is rather important for realization of an idea (innovation, project performance) [32]. In this social process, team members make exchanges and engage in social comparison, seeking to collaborate with others to solve the team's task problem and manage team conflict. The team might establish its own rules, values, and goals. When team members adopt the team's view and create a shared understanding of the cognitive process, these team values can guide the team process and outcomes directly. Collective team values direct members toward common goals. When members adopt shared goals, the team achieves high performance. These conditions influence members to generate more ideas, improve ideas or realize ideas within the team context. This study also echoes Nonaka and Takeuchi's findings that collectivism can enhance idea realization [32].

However, the effect of a single factor on team creativity (i.e., the effect of collectivistic value orientation on the social process; the interaction effect of team task proficiency on cognition and team vitality on motivation; 
and the effects of collectivistic value orientation on the social process and team vitality on the motivational process) is limited. The occurrence of three simultaneous, interactive team processes can enhance team creativity. Hence, the most representative model is that in which team task proficiency in the cognitive process promotes team vitality, which in turn has a motivation effect through collectivistic value orientation on social interaction and, through the effect of team vitality on the motivational process, enhances team creativity.

Finally, team task proficiency and team collective value can enhance team members' motivation in the motivational process. According to a comparison of intrinsic motivation and thriving, vitality was selected from among the crucial team member variables, and its effects on team task proficiency, collectivistic value orientation, and team creativity in the motivational process were examined. Vitality, being energized and alive at work, resembles intrinsic motivation, the psychological forces that stimulate behavior. Hence, team task proficiency in the cognitive process enhances team vitality in the motivational process through collectivistic value orientation in the social process because the interactive effects of collectivistic value orientation on the social process and team vitality on the motivational process are stronger than the effects of team task proficiency on the cognitive process and team vitality on the motivational process in the interaction. In addition to hypothesizing that vitality benefits team creativity according to the literature review, we determined how team task proficiency, collectivistic value orientation, and team vitality interact in the social, cognitive, and motivational processes and how they promote team creativity.

\section{Conclusions}

This study applied the model of creativity of [17] to identify crucial team member variables and analyze the effects of the variables on interactions among cognitive, social, and motivational processes and on the enhancement of team creativity. The results of this study showed that three crucial team member variable of team task proficiency, collectivistic value orientation, and team vitality positively influence team creativity both indirectly and directly. The study examined the mediating effect of team vitality on the relationship between collectivistic value orientation and team creativity and the mediating effect of collectivistic value orientation on the relationship between team task proficiency and team vitality. The results indicated that team task proficiency affected team vitality more through collectivistic value orientation and that team task proficiency and collectivistic value orientation affected team creativity more through team vitality. This outcome supports the study's argument that team vitality is a trigger of team creativity. In addition to demonstrating the value of team vitality, the study clarified how team task proficiency, collectivistic value orientation, and team vitality interact, contributing to creativity in the team process.

On the basis of the model of creativity of [17], this study clarified key team member variables that interact with cognitive, social, and motivational processes to enhance team creativity. This study's empirical results demonstrated a clear theoretical framework of the interactions among team task proficiency, collectivistic value orientation, team vitality, and team creativity, contributing new insights into team-level creative synergy and strategies for developing team creativity. The study provides a deeper understanding of creativity theory, because the analysis progressed from the individual to team levels, and of the team process in which team members contribute, combining relevant inputs, team task proficiency, collectivistic value orientation, and team vitality in an optimal fashion to yield team creativity. These results and theoretical framework will enrich future studies of team creativity and the team process.

The results showed that team vitality has as stronger effect on team creativity than other factors do. Team leaders or managers must incite passion among team members and motivate them to invest their efforts in work or team tasks through formal or informal methods. Stimulating team members' vitality raises their creativity and is beneficial for producing creative ideas that can be implemented as innovative practice.

After having stimulated their team members' vitality, team leaders and managers must still discuss, communicate, and coordinate with the team members regarding the values that embody their team because the study results indicate that collectivistic value orientation has a positive effect on team vitality. When the team members recognize and embody their team's values, team coherence that incites vitality in the team members is instilled. In turn, team vitality enhances team creativity. Therefore, team leaders and managers should invest more resources in developing team consciousness, cohesion, and collaboration to increase collectivistic value orientation. However, team task proficiency affected team vitality through collectivistic value orientation; team leaders and managers should not overemphasize improving team members' ability and expertise in team problem solving. A more effective approach for achieving superior performance in creativity and innovation might be to shift the attention from team task proficiency and rearrange or allocate more resources to improving collectivistic value orientation.

This study had certain limitations that can be used as a reference in designing future studies. First, the research subjects were members of project production teams in the undergraduate departments of science and technology universities that specialize in technical and vocational education services. The purpose of this study was to gain an empirical understanding of the creative performance of university students in the technical and vocational education system. Thus, future studies can address other emerging and traditional industries, such as the digital convergence, 
franchising, and cultural and creative industries, and conduct differentiated comparisons of creativity at multiple levels, providing an understanding of the operational frameworks and design requirements of creativity for practitioners and teams in various industries. Second, we selected only four variables (i.e., team task proficiency, collectivistic value orientation, team vitality, and team creativity) on the basis of the team-level collective cooperation process and the creativity and innovation topics that have been of interest to enterprises and vocational education services but did not include other possible mediating, moderating, or control variables for team creativity. Future studies could include other cognitive-social-motivational process variables, such as school and business environmental context variables, variables related to the teaching profession, external or internal motivational variables, and control variables (e.g., time pressure or creative task features), to improve the comprehensiveness of the team creativity framework. Third, we relied on questionnaires from a limited timeframe. Although we integrated the self-assessments from the student team members with assessments from observers to prevent common method variance, we did not implement a qualitative or longitudinal research design. Future studies could adopt a control design involving longitudinal studies and experimental research. Performance variations and changes in the mentality of students regarding team creativity during various learning phases could be investigated to understand the inner thoughts of the students toward the modes of operation used in collective collaboration and creation.

\section{Acknowledgements}

This study was made possible by a grant from the MOST sponsored study program received by the first author (grant number MOST102-2410- H-266-001-).

\section{REFERENCES}

[1] Amabile, T. M. (1996).Creativity in Context: Update to the Social Psychology of Creativity. Colorado: Westview Press.

[2] Amabile, T. M., Coon, H., Conit, R., Herron, M., \& Lazenby, J. (1996). Assessing the Work Environment for Creativity. Academy of Management Journal, 39 (5), 1154-1184. doi:10.2307/256995.

[3] Arbuckle, J. L. (2003). AMOS 5.0 Update to the AMOS User's Guide. Chicago: Small Waters Corporation.

[4] Armenakis, A. A., Feild, H. S., Giles, W. F., Hirschfeld, R. R., \& Jordan, M. H. (2006). Becoming Team Players: Team Members' Mastery of Teamwork Knowledge as a Predictor of Team Task Proficiency and Observed Teamwork Effectiveness. Journal of Applied Psychology, 91(2), 467-474. doi:10.1037/0021-9010.91.2.467

[5] Atwater, L., \& Carmeli, A. (2009). Leader-Member Exchange, Feelings of Energy, and Involvement in Creative Work. The Leadership Quarterly, 20 (3), 264-275. doi:10.1016/j.leaqua.2007.07.009

[6] Baas, M., De Dreu, C. K., \& Nijstad, B. A. (2008). Hedonic Tone and Activation Level in the Mood-Creativity Link: Toward a Dual Pathway to Creativity Model. Journal of Personality and Social Psychology, 94(5), 739-756. doi:10.1037/0022-3514.94.5.739

[7] Bechtoldt, M. N., Choi, H. S., \& Nijstad, B. A. (2012). Individuals in Mind, Mates by Heart: Individualistic Self-Construal and Collective Value Orientation as Predictors of Group Creativity. Journal of Experimental Social Psychology, 48(4), 838-844.doi:10.1016/j.jesp.2012.02.014

[8] Bechtoldt, M. N., Choi, H. S., De Dreu, C. K., \& Nijstad, B. A. (2010). Motivated Information Processing, Social Tuning and Group Creativity. Journal of Personality and Social Psychology, 99(4), 622-637.doi:10.1037/a0019386

[9] Bennett, J. M., Lawrence, R. H., Liang, J., \& Whitelaw, N. A. (1990). Appropriateness of Composites in Structural Equation Models. Journal of Gerontology, 45(2), S52-S59.doi:10.1093/geronj/45.2.S52

[10] Carmeli, A., \& Spreitzer, G. M. (2009). Trust, Connectivity, and Thriving: Implications for Innovative Behaviors at Work. The Journal of Creative Behavior, 43(3), 169-191.doi:10.1002/j.2162-6057.2009.tb01313.x

[11] Chen, G., \& Kanfer, R. (2006). Toward a Systems Theory of Motivated Behavior in Work Teams. Research in Organizational Behavior, 27, 223-267. doi:10.1016/S0191-3085(06)27006-0

[12] Choi, J. N., \&Sung, S. Y. (2012). Effects of Team Knowledge Management on the Creativity and Financial Performance of Organizational Teams. Organizational Behavior and Human Decision Processes, 118 (1), 4-13. doi:10.1016/j.obhdp.2012.01.001

[13] De Vreede, T., \& Reiter-Palmon, R., Wigert, B. (2012). Team Creativity and Innovation: The Effect of Group Composition, Social Processes, and Cognition. In M. Mumford (Ed.), Handbook of Organizational Creativity (pp. 295-326). London, England: Academic Press.

doi:10.1016/B978-0-12-374714-3.00013-6

[14] Deci, E. L., Manly, J. B., Nix, G. A., \& Ryan, R. M. (1999). Revitalization through Self-Regulation: The Effects of Autonomous and Controlled Motivation on Happiness and Vitality. Journal of Experimental Social Psychology, 35(3), 266-284.doi:10.1006/jesp.1999.1382

[15] Demaree, R. G., James, L. R., \& Wolf, G. (1993). $r_{w g}$ : An Assessment of Within-Group Interrater Agreement. Journal of Applied Psychology, 78 (2), 306-309. doi:10.1037/0021-9010.78.2.306

[16] Dutton, J., Grant, A. M., Sonenshein, S., Spreitzer, G., \& Sutcliffe, K. (2005). A Socially Embedded Model of Thriving at Work. Organization Science, 16 (5), 537-549. doi:10.1287/orsc. 1050.0153 
[17] Dzindolet, M., \& Paulus, P. B. (2008). Social Influence, Creativity and Innovation. Social Influence, 3 (4), 228-247. doi:10.1080/15534510802341082

[18] Fairchild, A. J., Fritz, M. S., \& MacKinnon, D. P. (2007). Mediation Analysis. Annual Review of Psychology, 58, 593-614. doi:10.1146/annurev.psych.58.110405.085542

[19] Garnett, F. G., Gibson, C., Spreitzer, G., \& Porath, C. (2012). Thriving at Work: Toward Its Measurement, Construct Validation, and Theoretical Refinement. Journal of Organizational Behavior, 33(2), 250-275. doi:10.1002/job.756

[20] George, J. M., \& Zhou, J. (2001). When Job Dissatisfaction Leads to Creativity: Encouraging the Expression of Voice. Academy of Management Journal, 44(4), 682-696. doi: $10.2307 / 3069410$

[21] Goh, K. T., Goodman, P. S., \& Weingart, L. R. (2013). Team Innovation Processes an Examination of Activity Cycles in Creative Project Teams. Small Group Research, 44(2), 159-194.doi:10.1177/1046496413483326

[22] Griffin, M. A., Neal, A., \& Parker, S. K. (2007). A New Model of Work Role Performance: Positive Behavior in Uncertain and Interdependent Contexts. Academy of Management Journal, 50(2), 327-347. doi:10.5465/AMJ.2007.24634438

[23] Griffin, R. W., Sawyer, J. E., \& Woodman, R. W. (1993). Toward a Theory of Organizational Creativity. Academy of Management Review, 18 (2), 293-321. doi:10.5465/AMR.1993.3997517

[24] Hancock, G. R., \& Nevitt, J. (2004). Evaluating Small Sample Approaches for Model Test Statistics in Structural Equation Modeling. Multivariate Behavioral Research, 39(3), 439-478.doi:10.1207/S15327906MBR3903 3

[25] Kanfer, R. (1990). Motivation and Individual Differences in Learning: an Integration of Developmental, Differential and Cognitive Perspectives. Learning and Individual Differences, 2(2), 221-239.doi:10.1016/1041-6080(90)90023-A

[26] Latham, G. P., \& Pinder, C. C. (2005). Work Motivation Theory and Research at the Dawn of the $21^{\text {st }}$ Century. Annual Review of Psychology, 56, 485-516. doi:10.1146/annurev.psych.55.090902.142105

[27] Lee, J. Y., MacKenzie, S. B., Podsakoff, N. P., \& Podsakoff, P. M. (2003). Common Method Biases in Behavioral Research: A Critical Review of the Literature and Recommended Remedies. Journal of Applied Psychology, 88(5), 879-903. doi:10.1037/0021-9010.88.5.879

[28] Lovelace, K., Shapiro, D. L., \& Weingart, L. R. (2001). Maximizing Cross-Functional New Product Teams' Innovativeness and Constraint Adherence: a Conflict Communications Perspective. Academy of management journal, 44(4), 779-793.doi:10.2307/3069415

[29] Luo, L., \& Zhou, C. (2012). Group Creativity in Learning Context: Understanding in a Social-Cultural Framework and Methodology. Creative Education, 3 (4), 392-399. doi:10.4236/ce.2012.34062

[30] Mathisen, G. E. (2011). Organizational Antecedents of Creative Self - Efficacy. Creativity and Innovation Management, 20 (3), 185-195. doi:10.1111/j.1467-8691.2011.00606.x

[31] McWilliam, E. (2009). Teaching for creativity: from sage to guide to meddler. Asia Pacific Journal of Education, 29(3), 281-293.

[32] Nonaka, I., \& Takeuchi, H. (1995). The Knowledge-creating Company: How Japanese Companies Create the Dynamics of Innovation. Oxford University Press: New York.

[33] Obstfeld, D.Sutcliffe, K. M., \& Weick, K. E., (1999). Organizing for High Reliability: Processes of Collective Mindfulness. In B. Staw \& L. L. Cummongs (Eds.), Research in Organizational Behavior, (Vol. 21, pp. 81-123). Greenwich, CT: JAI Press.

[34] Paulus, L. E., \& Paulus, P. B. (1997). Implications of Research on Group Brainstorming in Gifted Education. Roeper Review, 19(4), 225-229. doi:10.1080/02783199709553834

[35] Shalley, C. E., \&Zhou, J. (2007). The Handbook of Organizational Creativity. Boca Raton, FL: Taylor \& Francis Group.

[36] Shin, Y. (2014). Positive Group Affect and Team Creativity Mediation of Team Reflexivity and Promotion Focus. Small Group Research, 45 (3), 337-364 doi:10.1177/1046496414533618

[37] Small and Medium Enterprise Administration, Ministry of Economic Affairs. (2014). 2014 White Paper on Small and Medium Enterprises. Taipei City, Taiwan: Small and Medium Enterprise Administration, Ministry of Economic Affairs.

[38] Sonnenburg, S. (2004). Creativity in Communication: A Theoretical Framework for Collaborative Product Creation. Creativity and Innovation Management, 13(4), 254-262. doi:10.1111/j.0963-1690.2004.00314.x

[39] Taggar, S. (2002). Individual Creativity and Group Ability to Utilize Individual Creative Resources: A Multilevel Model. Academy of Management Journal, 45(2), 315-330.doi:10.2307/3069349

[40] Yeh, Y. M. (2011). A Study on the Relationship Model Among Organizational Justice, Quality of Work Life, Organizational Trust, Thriving, and Innovative Behavior in Innovative Incubation Enterprises (Unpublished Doctorate Thesis). Graduate Institute of Technological and Vocational Education, National Taipei University of Technology, Taipei City.

[41] Yeh, Y. M., \& Hsiang, W. H. (2014). Team vitality: One of the Forces to Trigger Group Creativity. Proceeding from 2014 2nd International Conference on Advanced Education Technology and Management Science (AETMS 2014), Hong Kong, China. 